\title{
A critical evaluation of the factors affecting the survival and persistence of beneficial bacteria in healthy adults
}

\author{
L. Morelli ${ }^{1 *}$ and P. Pellegrino ${ }^{2}$ \\ ${ }^{1}$ DISTAS - Faculty of Agriculture, Food and Environmental Sciences, Università Cattolica del Sacro Cuore, Via Emilia \\ Parmense 84, Piacenza, 29122, Italy; ${ }^{2}$ Sanofi Consumer Health Care, Milan, 20158, Italy; lorenzo.morelli@unicatt.it
}

Received: 9 February 2021 / Accepted: 17 April 2021

(c) 2021 Wageningen Academic Publishers

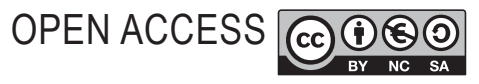

REVIEW ARTICLE

\begin{abstract}
The efficacy of a probiotic depends on its ability to survive and persist in the digestive tract. Regulatory agencies around the world recommend minimum dosages in order for a product to be termed a probiotic. However, the effect of dosage on the survival of the bacteria in the gut - the primary objective of probiotic administration - has not been critically evaluated. We performed a systematic literature review to assess the available data on the survival rate, during gastrointestinal transit, of probiotic bacteria that were orally administered to healthy adults. We also evaluated the persistence of the administered strain(s) after discontinuation of treatment and the potential role played by the food matrix in which probiotics have been administered. From a regulatory perspective, the profile of the target population is key to establishing the efficacy of probiotics. Therefore, we focussed on subjects without disease conditions. We evaluated 17 studies of single strains and 13 studies of multi-strain products, which reported survival and persistence outcomes. Persistence in the gut and recovery from stool were strain dependent. When the administered dose was higher than $10^{10} \mathrm{cfu} /$ day, the probiotic could be recovered from stool regardless of the strain used. Treatment duration did not affect faecal recovery. Thus, dosage recommendations for probiotics by regulatory agencies are lower than that required for a strain to survive, persist and be efficacious in the gut.
\end{abstract}

Keywords: gastrointestinal tract transit, multi-strain, single strain

\section{Introduction}

Between 2001 and 2002, an Expert Panel Consultation convened by the Food and Agriculture Organization (FAO) and the World Health Organization (WHO) established guidelines for the evaluation of health and nutritional properties of probiotics in food (FAO/WHO, 2001). These documents provided the widely accepted (Hill et al., 2014) definition of probiotics: 'live microorganisms that, when administered in adequate amounts, confer a health benefit on the host.'

However, these documents contain two additional, but less known, key statements (FAO/WHO, 2001):

1. 'For use in foods, probiotic microorganisms should not only be capable of surviving passage through the digestive tract, but also have the capability to proliferate in the gut.'
2. 'It is the ability to remain viable at the target site and to be effective, that should be verified for each potentially probiotic strain.'

The ability to survive and persist in the digestive tract forms the basis of the FAO/WHO guidelines. Regulatory agencies all over the world have used these statements as references. For instance, the European Food Safety Authority (EFSA, 2016), the US Food and Drug Administration (FDA), and Health Canada have used them as templates for their own guidelines on probiotics, as have agencies in China, India, Brazil, Argentina, and other nations. Some of these national guidelines have also recommended specific dosages for probiotics. Italy's Ministry of Health requires that a product termed 'probiotic' contain a minimum dose of $10^{9}$ viable bacteria/day (Ministero della Salute, 2013). In China, the State Administration for Market Regulation issued a document (CIRS, 2019) which stated that: 'The number of 
viable bacteria of each kind of probiotic health food during its shelf life shall not be less than $10^{6} \mathrm{cfu} / \mathrm{ml}(\mathrm{g})$.' However, the rationale behind these dosage recommendations has not been fully justified through empirical studies.

Despite extensive research on the health benefits of probiotic bacteria (Sánchez et al., 2017; Sanders et al., 2019; Szajewska and Hojsak, 2020), there is no consensus on the dose to be administered that would allow the establishment and persistence of these strains in the gut after treatment has ended.

The potential relationship between the administered dose and physiological effects of probiotics has been described previously (Ouwehand, 2017). However, this review lacked a critical evaluation of the reported survival/persistence rates of the administered bacteria and the observed probiotic outcomes - a 'dose-response' evaluation. The review also focused on clinical effects in subjects who had underlying pathological conditions.

In recent years, studies have tested the efficacy and safety of 'modified' probiotics, such as those that have been heat-killed or those that contain substances secreted by probiotics (termed 'postbiotics'). Evidence for their efficacy is still scarce in human trials. A systematic review found that in the majority of trials conducted to date, 'modified' probiotics were not significantly different from conventional probiotics in terms of efficacy, and were advantageous only in two specific disease states: adult obesity and chronic diarrhoea (Zorzela et al., 2017).

In this review, we focus on the ability of viable probiotic strains to survive and reproduce in the gastrointestinal tract of healthy adults. We summarise and evaluate the available data on dose-response outcomes of clinical studies. Results from in vitro studies or animal models, and studies conducted in paediatric subjects or those fed with infant formula will not be discussed here.

\section{Materials and methods}

To find relevant literature, a PubMed and Scopus search was performed using 'probiotics' and 'humans' as the main key words, combined with 'survival' and 'persistence' in two waves of search for each database. The filter 'clinical trial' was also applied in a further wave of search; however, results obtained without this filter were also retained for further analyses. Only manuscripts published in English as full texts in peer reviewed journals were retained.

Manuscripts dealing with non-prokaryotic probiotics, such as yeast, were excluded from this review. Because the scope of this review was limited to healthy subjects, all data obtained from clinical studies involving subjects with pathological conditions were discarded. Studies in which probiotics were co-administered with prebiotics and those that did not identify the strain of the probiotic were excluded. Studies that did not clearly describe the treatment regimen (dose or length of treatment) were also omitted. The retrieved publications were then classified into those dealing with a single strain and those dealing with multi-strain products.

Doses are presented as colony forming units (cfu), and represent cfu/day. For practical reasons, the new taxonomic nomenclature of the Lactobacillus genus (Zheng et al., 2020) has not been used; instead, we use the old genus names.

\section{Results}

The literature search in each database using the relevant keywords yielded a large number of studies (Table 1 shows the output after removing duplicate results). After independent checking of the abstracts, we selected 31 studies, reported in 28 publications, for the analysis. Two publications (Plaza-Diaz et al., 2013; Prilassnig et al., 2007), which report data on single and multi-strain products, were considered in both categories. Most studies expressed faecal recovery and persistence as either cfus or the number of subjects testing positive for the strain.

\section{Identification of probiotic strains in faecal samples}

Most studies used strain-specific methods to identify the administered probiotic strain in faeces. The most common methods included strain-specific PCR and rapid amplification of polymorphic DNA (RAPD) genotyping (Arioli et al., 2018; Dommels et al., 2009; Ghelardi et al., 2015; Hutt et al., 2011; Mai et al., 2017; Matto et al., 2006; Plaza-Diaz et al., 2013; Prilassnig et al., 2007; Rattanaprasert et al., 2014; Saxelin et al., 2010; Taverniti et al., 2019).

Saxelin et al. (1995) and Alander et al. (1999) identified Lactobacillus rhamnosus GG by colony morphology and the strain's inability to ferment lactose. Four studies employed strain-specific probes in colony hybridisation or dot-blot hybridisation assays (De Champs et al., 2003; DonnetHughes et al., 1999; Shinoda et al., 2001; Wassenaar et al., 2014). Vesa et al. (2000) used mutant strains resistant to rifampicin and streptomycin, with fermentation profiles

Table 1. Results of the literature search, using the keywords 'survival' and 'persistence' with the filter 'clinical trials', to retrieve studies dealing with faecal recovery of probiotics.

$\begin{array}{llll} & \text { PubMed } & \text { Scopus } & \begin{array}{l}\text { Applying the filter } \\ \text { 'clinical trials' }\end{array} \\ \text { Survival } & 1,970 & 1,298 & 158 \\ \text { Persistence } & 494 & 176 & 89\end{array}$


identical to those of the original strains; this allowed their detection on De Man, Rogosa and Sharpe (MRS) media containing these antibiotics.

Larsen et al. (2006) also used MRS media with antibiotics specific to Bifidobacterium animalis subsp. lactis BB-12 and Lactobacillus paracasei subsp. paracasei (CRL-431). They confirmed the identity of the resultant colonies using pulsed-field gel electrophoresis and fluorescent whole cell hybridisation. Lund et al. (2002) used pulsed-field gel electrophoresis to compare banding patterns between the Enterococcus faecium strain from the Causido ${ }^{\circledR}$ culture that they administered and those that they isolated from faeces. Jacobsen et al. (1999) and Klingberg and Budde (2006) used strain-specific Internal Transcribed Spacer region (ITS)-PCR and restriction enzyme analysis followed by pulsed-field gel electrophoresis to identify the probiotic strains they isolated in their studies.

Khine et al. (2019) used a strain-specific monoclonal antibody in an enzyme-linked immunosorbent assay (ELISA) to detect Lactobacillus casei Shirota. Van Bokhorst-Van de Veen et al. (2012) amplified and sequenced a variable intergenic region to distinguish between the probiotic strains isolated from faeces samples. Five studies did not conduct strainlevel testing to identify the administered probiotics in faeces (Ahmed et al., 2007; Hanifi et al., 2015; Plaza-Diaz et al., 2013; Rochet et al., 2008; Smith et al., 2011).

\section{Survival and persistence of probiotics administered as a single strain}

We retrieved 17 publications reporting survival and/or persistence outcomes of clinical studies performed in a total of 432 healthy humans with single strains (mainly belonging to Lactobacillus or Bifidobacterium genera; Table 2). Of these, 19 subjects from two studies participated in cross-over feeding studies (De Champs et al., 2003; Prilassnig et al., 2007). Five studies reported dose-response measurements for a total of 195 subjects (Ahmed et al., 2007; De Champs et al., 2003; Donnet-Hughes et al., 1999; Hanifi et al., 2015; Saxelin et al., 1995). One study involved administration of the same dosage daily or on alternate days to nine subjects each (Smith et al., 2011).

Among the single-strain studies, the administered daily dose ranged between $10^{7} \mathrm{cfu}$ and $10^{12} \mathrm{cfu}$ and faecal recovery ranged between 0 and $10^{12} \mathrm{cfu}$ (Table 2). Among studies that conducted strain-specific identification, recovery during treatment ranged between 0 and $10^{8} \mathrm{cfu}$. Probiotic strains were successfully recovered from stool during the treatment period in all studies, with the exception of three out of the six products tested by Prilassnig et al. (2007). Of these, two products were single strain probiotics: L. casei var. rhamnosus (Antibiophilus ${ }^{\oplus}$ ) and L. case $i$ Immunitas $\left(\right.$ Actimel $\left.^{\odot}\right)$.
Among publications reporting dose responses, two studies dealing with lactobacilli (Donnet-Hughes et al., 1999; Saxelin et al., 1995) found a linear relationship between dosage and persistence during treatment (Table 2). A study (Hanifi et al., 2015) that focused on the spore former, Bacillus subtilis RO179, reported a linear relationship between the administered doses $\left(10^{8}, 10^{9}\right.$, and $\left.10^{10} \mathrm{cfu}\right)$ and faecal recovery.

Lactobacillus strains appear to persist in the intestines over a maximum length of two weeks (Alander et al., 1999) after the cessation of probiotics, with most being recovered up to 5-7 days after treatment (Table 2). A notable exception is $L$. casei Lcr35, which was observed three weeks after treatment ended (De Champs et al., 2003). The administered probiotic strains did not persist in $100 \%$ of treated subjects in any of the studies considered.

\section{Survival and persistence of multi-strain probiotics}

We retrieved 13 publications reporting clinical studies, performed in 260 healthy adults, which recorded the survival and/or persistence outcomes of a mixture of strains, mainly belonging to Lactobacillus or Bifidobacterium genera (Table 3). Of these, 44 subjects participated in cross-over feeding studies (Ghelardi et al., 2015; Klingberg and Budde, 2006; Prilassnig et al., 2007). Several authors reported presence and persistence for only some of the strains that they administered (Table 3).

Three studies used a mixture of different Lactobacillus strains (Hutt et al., 2011; Klingberg and Budde, 2006; Van Bokhorst-Van de Veen et al., 2012), while six others used a mixture of Lactobacillus and Bifidobacterium (Larsen et al., 2006; Mai et al., 2017; Matto et al., 2006; Plaza-Diaz et al., 2013; Prilassnig et al., 2007; Taverniti et al., 2019). Saxelin et al. (2010) used a mixture containing more than two genera, whereas Wassenaar et al. (2014) used a mixture of six strains of Escherichia coli and Ghelardi et al. (2015) used four strains of Bacillus clausii. The daily administered doses ranged from $10^{7}$ to $10^{11} \mathrm{cfu}$. Treatment duration varied from one-shot treatment (Ghelardi et al., 2015; Wassenaar et al., 2014) to continuous treatment over 30 days (Plaza-Diaz et al., 2013). Faecal recovery ranged between $10^{3}$ and $10^{9} \mathrm{cfu}$.

In a study dealing with four strains of B. clausii (Ghelardi et al., 2015), 20 volunteers were given one of two formulations (vials and capsules; ten per arm) of the same probiotic at the same dosage $\left(10^{9} \mathrm{cfu} /\right.$ day), to determine the bioequivalence of the formulations. In a unique approach, the authors calculated the total amount of each of the four strains (taking into consideration the total faecal output) excreted by each volunteer after the one-shot administration through vials or capsules. The probiotic was recovered from faeces in both study arms, up to 12 days after the single administration. Thus, the formulation had little to no effect on the recovery 
Table 2. Seventeen studies reporting survival and/or persistence outcomes of clinical studies using single strains in healthy subjects.

\begin{tabular}{|c|c|c|c|c|c|c|}
\hline Strain & $\begin{array}{l}\text { Daily dose(s) in cfu } \\
\text { unless otherwise } \\
\text { mentioned }\end{array}$ & $\begin{array}{l}\text { Treatment } \\
\text { (days) }\end{array}$ & Subjects & $\begin{array}{l}\text { Recovery during } \\
\text { treatment }\end{array}$ & $\begin{array}{l}\text { Persistence after } \\
\text { treatment }\end{array}$ & Reference \\
\hline $\begin{array}{l}\text { Lactobacillus } \\
\text { rhamnosus GG }\end{array}$ & $\begin{array}{l}\text { dose A: } 1.6 \times 10^{8} \\
\text { dose } B: 1.2 \times 10^{10} \\
\text { gelatin capsule }\end{array}$ & 7 & 20 & $\begin{array}{l}10^{5} \text { to } 10^{6} \text { for dose } \\
B ; 10^{3} \text { in one } \\
\text { sample for dose } A\end{array}$ & $\mathrm{NA}^{\mathrm{a}}$ & Saxelin et al., 1995 \\
\hline $\begin{array}{l}\text { Lactobacillus johnsonii } \\
\text { La1 }\end{array}$ & $\begin{array}{l}\text { low dose: approx. } 10^{8} \\
\text { high dose: approx. } 10^{9}\end{array}$ & $21+$ follow up & 42 & $\begin{array}{l}\text { low dose: } 10^{4} \text {; high } \\
\text { dose: } 10^{5}\end{array}$ & $\begin{array}{l}\text { negative detection } \\
7 \text { days after end } \\
\text { of treatment }\end{array}$ & $\begin{array}{l}\text { Donnet-Hughs et } \\
\text { al., } 1999\end{array}$ \\
\hline L. rhamnosus GG & $6 \times 10^{10}$ & $12+$ follow up & 21 & $\begin{array}{l}\text { strain was detected } \\
\text { in all volunteers at } \\
\text { approx. } 10^{6} \mathrm{cfu}\end{array}$ & $\begin{array}{l}8 \text { out of } 21 \text { positive } \\
\text { one week after } \\
\text { discontinuation at } \\
10^{3}-10^{5} \mathrm{cfu}\end{array}$ & Alander et al., 1999 \\
\hline $\begin{array}{l}\text { Lactobacillus } \\
\text { fermentum KLD, } \\
\text { Lactobacillus } \\
\text { plantarum NCIMB } \\
8826, \text { Lactococcus } \\
\text { lactis MG } 1363\end{array}$ & $\begin{array}{l}10^{8} \text { of } L \text {. plantarum in } \\
\text { fermented milk }\end{array}$ & $\begin{array}{l}7+21 \text { follow } \\
\text { up }\end{array}$ & $\begin{array}{l}18 \text { (6 with } \\
\text { L. plantarum) }\end{array}$ & $\begin{array}{l}\text { L. plantarum } \\
\text { NCIMB 8826: } 10^{8} \text {; } \\
\text { the other strains } \\
\text { were not tested }\end{array}$ & $\begin{array}{l}\text { not detected in } \\
\text { faeces two weeks } \\
\text { after treatment } \\
\text { ended }\end{array}$ & Vesa et al., 2000 \\
\hline $\begin{array}{l}\text { Enterococcus faecium } \\
\text { Causido }^{\circ}\end{array}$ & $4.5-7.5 \times 10^{9}$ & 10 & $\begin{array}{l}10 \text { (+10 receiving } \\
\text { concomitant } \\
\text { vancomycin) }\end{array}$ & $\begin{array}{l}10^{3}-10^{6} \text { cfu } \\
\text { (8/10 positive) }\end{array}$ & $\begin{array}{l}\text { all subjects } \\
\text { negative } 3 \text { weeks } \\
\text { after treatment } \\
\text { ended }\end{array}$ & Lund et al., 2002 \\
\hline $\begin{array}{l}\text { Lactobacillus helveticus } \\
\text { CP53 }\end{array}$ & one shot $10^{11}$ cells & 5 & 7 & $\begin{array}{l}10^{4}-10^{8} \text { cfu in } 4 / 7 \\
\text { subjects }\end{array}$ & $N A^{a}$ & Shinoda et al., 2001 \\
\hline $\begin{array}{l}\text { Lactobacillus casei } \\
\text { subsp. rhamnosus } \\
\text { Lcr35 }\end{array}$ & $\begin{array}{l}\text { dose } A: 10^{8} ; \text { dose } \mathrm{B}: \\
10^{10} ; \text { dose C: } 10^{12}\end{array}$ & $7+7$ & 12 (cross-over) & $\begin{array}{l}10^{4} / 10^{5} \text { (not related } \\
\text { to the dose) }\end{array}$ & $\begin{array}{l}10^{3}-10^{6} \text { at seven } \\
\text { days after } \\
\text { treatment ended }\end{array}$ & $\begin{array}{l}\text { De Champs et al., } \\
2003\end{array}$ \\
\hline $\begin{array}{l}\text { Bifidobacterium lactis } \\
\text { HN019 }\end{array}$ & $\begin{array}{l}\text { high: } 5 \times 10^{9} \text {; medium: } \\
1 \times 10^{9} \text {; low: } 6.5 \times 10^{7}\end{array}$ & 28 & $\begin{array}{l}80 \text { (20 in each } \\
\text { dosage }+ \\
20 \text { placebo) }\end{array}$ & $\begin{array}{l}\text { high: } 10^{9} \text {; medium: } \\
\text { 109; low: } 10^{9} \mathrm{~b} \text {; } \\
\text { (total bifidobacteria } \\
\text { levels) }\end{array}$ & $N A^{a}$ & Ahmed et al., 2007 \\
\hline $\begin{array}{l}\text { L. casei var. rhamnosus } \\
\text { (Antibiophilus }^{\odot} \text { ), } \\
\text { L. casei Immunitas } \\
\text { (Actimel }^{\odot} \text { ) }\end{array}$ & $\begin{array}{l}1 \times 10^{9} \text { cells } \\
\text { (Antibiophilus), } 1 \times 10^{10} \\
\text { cells (Actimel); each } \\
\text { product administered } \\
\text { separately }\end{array}$ & 7 & 7 (cross-over) & $\begin{array}{l}\text { no recovery in } 5 \\
\text { subjects for each } \\
\text { product }\end{array}$ & $N A^{a}$ & $\begin{array}{l}\text { Prilassnig et al., } \\
2007\end{array}$ \\
\hline $\begin{array}{l}\text { L. rhamnosus GG, } \\
\text { Lactobacillus reuteri } \\
\text { DSM } 17938\end{array}$ & $\begin{array}{l}1 \times 10^{9} \text { L. reuteri } 17938 \text {, } \\
5 \times 10^{9} \text { L. rhamnosus } \\
\text { GG; each strain } \\
\text { administered } \\
\text { separately }\end{array}$ & 21 & 29 & $\begin{array}{l}\text { In the range of } \\
10^{6} \text { per strain }\end{array}$ & $N A^{a}$ & $\begin{array}{l}\text { Dommels et al., } \\
2009\end{array}$ \\
\hline L. casei DN-114 001 $1^{\text {if }}$ & approx. $10^{10}$ & 8 & 7 & $\begin{array}{l}\text { Bacteria- } \\
\text { equivalents of } \\
\text { L. paracasei group } \\
2 \text { log higher than } \\
\text { at the start }\end{array}$ & $\begin{array}{l}\text { Recovery up to } 3 \\
\text { days; absence } \\
\text { after } 7 \text { days }\end{array}$ & Rochet et al., 2008 \\
\hline
\end{tabular}


Table 2. Continued.

\begin{tabular}{|c|c|c|c|c|c|c|}
\hline Strain & $\begin{array}{l}\text { Daily dose(s) in cfu } \\
\text { unless otherwise } \\
\text { mentioned }\end{array}$ & $\begin{array}{l}\text { Treatment } \\
\text { (days) }\end{array}$ & Subjects & $\begin{array}{l}\text { Recovery during } \\
\text { treatment }\end{array}$ & $\begin{array}{l}\text { Persistence after } \\
\text { treatment }\end{array}$ & Reference \\
\hline L. reuteri DSM 17938 & $10^{9}$ & $\begin{array}{l}\text { A: } 7 \text { every } \\
\text { day; B: } 7 \\
\text { on alternate } \\
\text { days }\end{array}$ & A: 9; B: 9 & $\begin{array}{l}\text { approx. } 10^{5} \text { in all } \\
\text { subjects }\end{array}$ & $\begin{array}{l}\text { A: } 4 / 9 \text { and } 2 / 9 \\
\text { positive after } \\
1 \text { and } 2 \text { weeks, } \\
\text { respectively; } \\
\text { B: } 3 / 9 \text { and } 2 / 9 \\
\text { positive after } \\
1 \text { and } 2 \text { weeks, } \\
\text { respectively }\end{array}$ & Smith et al., 2011 \\
\hline L. reuteri DSM 17938 & $5 \times 10^{8}$ & $7^{c}$ & 15 & $10^{8}$ & $\begin{array}{l}\text { after } 2 \text { days: } 10^{7} \text {; } \\
\text { after } 4 \text { days: } \\
\text { not detectable }\end{array}$ & $\begin{array}{l}\text { Rattanaprasert et } \\
\text { al., } 2014\end{array}$ \\
\hline $\begin{array}{l}\text { L. paracasei CNCM } \\
\text { I-4034; L. rhamnosus } \\
\text { CNCM I-4036; } \\
\text { Bifidobacterium breve } \\
\text { CNCM I-4035 }\end{array}$ & $9 \times 10^{9}$ & 30 & $\begin{array}{l}100 \text { total } \\
\text { (60 receiving } \\
\text { single strain, } \\
20 \text { multistrain, } \\
\text { and } 20 \text { placebo) }\end{array}$ & $\begin{array}{l}\text { not identified at } \\
\text { the strain level }\end{array}$ & $\begin{array}{l}\text { only L. rhamnosus } \\
\text { CNCM I-4036 } \\
\text { plate counted and } \\
\text { identified } 14 \text { days } \\
\text { after treatment }\end{array}$ & $\begin{array}{l}\text { Plaza-Diaz et al., } \\
2013\end{array}$ \\
\hline Bacillus subtilis R0179 & $\begin{array}{l}\text { dose } A: 0.1 \times 10^{9} \\
\text { dose } B: 1.0 \times 10^{9} \\
\text { dose } \text { C: } 10 \times 10^{9}\end{array}$ & 28 & 81 (20 placebo) & $\begin{array}{l}\text { dose } A: 10^{4} \\
\text { dose } B: 10^{5} \\
\text { dose } C: 10^{6} \mathrm{~b}\end{array}$ & $\begin{array}{l}\text { after } 7 \text { days: dose } \\
\text { B: } 10^{0.8} \text {; dose C: } \\
10^{2.1}\end{array}$ & Hanifi et al., 2015 \\
\hline $\begin{array}{l}\text { L. paracasei CNCM } \\
\text { I-1572 (DG) }\end{array}$ & $8 \times 10^{9}$ & 7 & 20 & $\begin{array}{l}10^{3.6}-10^{6.7} \mathrm{cfu} / \mathrm{g} \text { in } \\
\text { all volunteers }\end{array}$ & $\max .5$ days & Arioli et al., 2018 \\
\hline $\begin{array}{l}\text { L. casei Shirota } \\
\left(\text { YAKULT }^{\odot}\right)\end{array}$ & $1.0 \times 10^{10}$ & 14 & 21 & $\begin{array}{l}\text { day } 7: 10^{7} ; \text { day } 14: \\
10^{7} \mathrm{~b}\end{array}$ & $\begin{array}{l}\text { no positive subjects } \\
\text { after } 14 \text { days }\end{array}$ & Khine et al., 2019 \\
\hline
\end{tabular}

rate of the strains. There were more volunteers from whom faecal recovery was equal to or higher than the administered dose, and fewer volunteers in whom recovery was lower than the administered dose. Thus, the probiotic survives passage through the gastrointestinal tract. In particular, strain $\mathrm{O} / \mathrm{C}$ exhibited the potential to multiply in the gut: ten volunteers, irrespective of the study arm, had higher spore recovery than ingestion. Five volunteers showed the same behaviour for the strains N/R and four volunteers for strain SIN. None of the volunteers showed an increase in the number of recovered $\mathrm{T}$ strain when compared to administered dose. The predominant strain in both formulations, N/R, displayed the longest persistence.

We retrieved three dose response studies (Larsen et al., 2006; Taverniti et al., 2019; Van Bokhorst-Van de Veen et al., 2012), which deal with different formulations (capsules, yoghurt, cheese, and encapsulated cells), dosages, and treatment times. The faecal recovery of B. animalis subsp. lactis BB-
12 increased significantly $(P=0.001)$ with an increase in dose and persisted in a small number of subjects beyond the treatment period (Larsen et al., 2006). The analysis reported by Van Bokhorst-Van de Veen and colleagues (2012) did not allow them to conclude on the effect of the dosage of probiotics. However, Taverniti and colleagues (2019) identified a clear dose-dependence between the amount of recovered strains (one Bifidobacterium and three Lactobacillus strains) and the administered dosages. The higher dosage resulted in the recovery of $10^{6}$ to $10^{9} \mathrm{cfu} / \mathrm{g}$ faeces of the probiotic in all twenty subjects.

Persistence beyond treatment of multi-strain probiotics closely resembled that of single-strain products, ranging from three days (Hutt et al., 2011) to 17 days (Saxelin et al., 2010). Not all treated subjects had persistent strains in stool, with the notable exception of the mix used by Taverniti $e t$ al. (2019), in which all three strains were recovered from subjects administered the higher dose. 
Table 3. Thirteen studies reporting survival and/or persistence outcomes of clinical studies using multi-strain products in healthy subjects.

\begin{tabular}{|c|c|c|c|c|c|c|}
\hline Strains & Daily dose(s) in cfu & $\begin{array}{l}\text { Treatment } \\
\text { (days) }\end{array}$ & $\begin{array}{l}\text { Treated } \\
\text { subjects }\end{array}$ & $\begin{array}{l}\text { Recovery during } \\
\text { treatment }\end{array}$ & $\begin{array}{l}\text { Persistence after } \\
\text { treatment }\end{array}$ & Reference \\
\hline $\begin{array}{l}\text { Lactobacillus rhamnosus } \\
\text { 19070-2, Lactobacillus } \\
\text { reuteri DSM 12246, } \\
\text { L. rhamnosus LGG, } \\
\text { Lactobacillus delbrueckii } \\
\text { subsp. lactis CHCC } \\
\text { 2329, Lactobacillus casei } \\
\text { subsp. alactus CHCC } \\
3137\end{array}$ & $\begin{array}{l}10^{10} \text { twice a day of either } \\
\text { Mix } 1 \text { (L. rhamnosus } \\
\text { 19070-2 and L. reuteri } \\
\text { DSM 12246) or Mix } 2 \\
\text { (L. rhamnosus LGG, } \\
\text { L. delbrueckii subsp. } \\
\text { lactis CHCC } 2329 \text { and } \\
\text { L. casei subsp. alactus } \\
\text { CHCC } 3137 \text { ) }\end{array}$ & 18 & 12 & $\begin{array}{l}10^{5} \text { to } 10^{8} \text { cells/g in } \\
\text { some subjects }\end{array}$ & $\begin{array}{l}\text { L. reuteri DSM } 12246 \\
\text { (in one case) and } \\
\text { L. rhamnosus 19070-2 } \\
\text { and L. rhamnosus } \\
\text { GG (two cases each) } \\
\text { reisolated at five days } \\
\text { after treatment ended }\end{array}$ & $\begin{array}{l}\text { Jacobsen et } \\
\text { al., } 1999\end{array}$ \\
\hline $\begin{array}{l}\text { Bifidobacterium animalis } \\
\text { subsp. lactis BB-12); } \\
\text { L. paracasei subsp. } \\
\text { paracasei CRL-431) }\end{array}$ & $\begin{array}{l}\text { A: } 10^{8} ; B: 10^{9} ; C: 10^{10} \\
\text { D: } 10^{11} ; E: \text { placebo }\end{array}$ & $\begin{array}{l}21+14 \\
\text { follow up }\end{array}$ & $\begin{array}{l}75 \text { (15 in } \\
\text { each group) }\end{array}$ & $\begin{array}{l}\text { CRL-431 never } \\
\text { isolated. BB-12 } \\
\text { recovery strongly } \\
\text { dose-dependent: } \\
\text { A: 3/14; B: 7/15; C: } \\
\text { 7/15; D: } 13 / 15\end{array}$ & $\begin{array}{l}\text { B: } 1 / 15 ; \mathrm{D}: 2 / 15 \text { positive } \\
\text { at the end of the wash } \\
\text { out period; remaining } \\
\text { groups: } 0 / 15\end{array}$ & $\begin{array}{l}\text { Larsen et al., } \\
2006\end{array}$ \\
\hline $\begin{array}{l}3 \text { Lactobacillus plantarum } \\
\text { strains (MF1291, } \\
\text { MF1298, DC13), } \\
\text { Lactobacillus pentosus } \\
\text { MF1300, Lactobacillus } \\
\text { salivarius DC5 }\end{array}$ & $\begin{array}{l}\text { Mix A: MF1291 + MF1298; } \\
\text { Mix B: MF1300 + DC5 } \\
\text { + DC13; MF1298 in a } \\
\text { fermented sausage; } \\
6 \times 10^{9} \text { per strain, twice } \\
\text { a day }\end{array}$ & $\begin{array}{l}18+12 \\
\text { follow up }\end{array}$ & 17 cross-over & $\begin{array}{l}\text { MF1298: } 4 / 17 \\
\text { DC13: } 3 / 17 \\
\text { subjects in the } \\
\text { range } 10^{3}-10^{8} \\
\text { cfu/g }\end{array}$ & $\begin{array}{l}\text { MF1298 present in } 1 \\
\text { subject } 6 \text { days after } \\
\text { end of treatment; } \\
\text { DC13 present in } 1 \\
\text { subject } 6 \text { and } 12 \text { days } \\
\text { after end of treatment }\end{array}$ & $\begin{array}{r}\text { Klingberg and } \\
\text { Budde, } 2006\end{array}$ \\
\hline $\begin{array}{l}\text { L. paracasei F19, } \\
\text { Lactobacillus } \\
\text { acidophilus NCFB 1748, } \\
\text { Bifidobacterium animalis } \\
\text { subsp. lactis BB-12 } \\
\text { (Dofilus ABC yogurt) }\end{array}$ & $2 \times 10^{10}$ per strain & $\begin{array}{l}10+\text { up to } 19 \\
\text { follow up }\end{array}$ & 14 & $\begin{array}{l}\text { F19: } 14 / 14\left(10^{5.5}\right) ; \\
\text { BB-12: } 11 / 14 \\
\left(10^{6.9}\right) ; \text { NCFB 1748: } \\
3 / 14\left(10^{5.2}\right)^{\text {a }}\end{array}$ & $\begin{array}{l}\text { F19: } 2 / 10 \text { after } 4 \text { days } \\
\left(10^{4}-10^{5}\right) ; \text { BB-12: } \\
\text { 4/10 after } 4 \text { days }\left(10^{6}\right) ; \\
\text { 1/10 after } 9 \text { days }\left(10^{4}\right) ; \\
\text { 1/5 after } 19 \text { days }\left(10^{7}\right) ; \\
\text { NCFB } 1748: 1 / 14 \text { after } \\
4 \text { days }\left(10^{4.9}\right) ; 2 / 10 \\
\text { after } 9 \text { days }\left(10^{5}\right)\end{array}$ & $\begin{array}{l}\text { Matto et al., } \\
2006\end{array}$ \\
\hline $\begin{array}{l}\text { L. acidophilus combined } \\
\text { with Bifidobacterium } \\
\text { infantis (Infloran }{ }^{\odot} \text {, } \\
\text { Lactobacillus gasseri } \\
\text { combined with } \\
\text { Bifidobacterium longum } \\
\text { (Omniflora }^{\odot} \text { ) }\end{array}$ & $\begin{array}{l}\text { Infloran: } 10^{9} \text { per strain; } \\
\text { Omniflora: } 10^{8} \text { per strain }\end{array}$ & 7 & 7 cross-over & $\begin{array}{l}1 / 6 \text { positive subject } \\
\text { for Infloran only } \\
\text { at the end of the } \\
\text { treatment }\end{array}$ & $N^{b}$ & $\begin{array}{l}\text { Prilassnig et } \\
\text { al., } 2007\end{array}$ \\
\hline $\begin{array}{l}\text { L. rhamnosus GG and } \\
\text { LC705, B. animalis } \\
\text { subsp. lactis BB-12, } \\
\text { Propionibacterium } \\
\text { freudenreichii subsp. } \\
\text { shermanii JS }\end{array}$ & $\begin{array}{l}\text { comparison among } \\
3 \text { matrices: yoghurt, } \\
\text { cheese, capsules; } \\
\text { total cfu for the } 3 \text { strains: } \\
10^{10}\end{array}$ & $\begin{array}{l}14+21 \\
\text { follow up }\end{array}$ & 36 & $\begin{array}{l}\text { all strains detected } \\
\text { as genome copies } \\
\text { at the end of } \\
\text { treatment }\end{array}$ & $\begin{array}{l}\text { median number of } \\
\text { days after treatment } \\
\text { when each strain was } \\
\text { detected: LGG: 17; } \\
\text { LC705: 5; BB-12: 7; } \\
\text { PJS: } 7\end{array}$ & $\begin{array}{l}\text { Saxelin et al., } \\
2010\end{array}$ \\
\hline $\begin{array}{l}\text { L. gasseri } 177 \text { and E16B7, } \\
\text { L. fermentum } 338-1-1 \text {, } \\
\text { L. acidophilus } 821-3 \text {, } \\
\text { L. paracasei } 317\end{array}$ & $10^{10}$ per strain & $\begin{array}{l}5+15 \text { follow } \\
\text { up }\end{array}$ & 14 & $\begin{array}{l}\text { all strains recovered } \\
\text { at the end of the } \\
\text { treatment at about } \\
10^{6} \mathrm{cfu} / \mathrm{g} \text { each }\end{array}$ & $\begin{array}{l}\text { L. acidophilus } 821-3 \\
\text { was detected in } 4 / 9 \\
\text { subjects three days } \\
\text { after treatment ended } \\
\left(10^{4.3} \text { to } 10^{7} \mathrm{cfu} / \mathrm{g}\right) \text { and } \\
\text { in } 2 / 9 \text { subjects five } \\
\text { days after treatment } \\
\text { ended ( } 10^{8.3} \text { and } 10^{3.9} \\
\text { cfu/g) }\end{array}$ & $\begin{array}{l}\text { Hutt et al., } \\
2011\end{array}$ \\
\hline
\end{tabular}


Table 3. Continued.

\begin{tabular}{|c|c|c|c|c|c|c|}
\hline Strains & Daily dose(s) in cfu & $\begin{array}{l}\text { Treatment } \\
\text { (days) }\end{array}$ & $\begin{array}{l}\text { Treated } \\
\text { subjects }\end{array}$ & $\begin{array}{l}\text { Recovery during } \\
\text { treatment }\end{array}$ & $\begin{array}{l}\text { Persistence after } \\
\text { treatment }\end{array}$ & Reference \\
\hline $\begin{array}{l}21 \text { strains of } L \text {. plantarum } \\
\text { in mixes containing } 10 \\
\text { strains each }\end{array}$ & $\begin{array}{l}10^{11} \text { total amount of each } \\
\text { mixture; the WCFS1 } \\
\text { strain was included as a } \\
10 \text {-fold dilution series in } \\
\text { four steps }\end{array}$ & $\begin{array}{l}10+21 \\
\text { follow up }\end{array}$ & 10 & $\begin{array}{l}\text { One strain } \\
\text { (WCFS1) identified } \\
\text { by RT-PCR after } \\
7 \text { days }\end{array}$ & Only genomic detection & $\begin{array}{l}\text { Van Bokhorst- } \\
\text { Van de Veen } \\
\text { et al., } 2012\end{array}$ \\
\hline $\begin{array}{l}\text { L. paracasei CNCM } \\
\text { I-4034, Bifidobacterium } \\
\text { breve CNCM I-4035, L. } \\
\text { rhamnosus CNCM I-4036 }\end{array}$ & $9 \times 10^{9}$ per strain & $\begin{array}{l}30+15 \\
\text { follow up }\end{array}$ & 20 & $\begin{array}{l}\text { isolates not } \\
\text { identified at the } \\
\text { strain level }\end{array}$ & $\begin{array}{l}\text { L. rhamnosus CNCM } \\
\text { I-4036; recovered from } \\
\text { stools at day } 30 \text { (level } \\
\text { of presence unknown) }\end{array}$ & $\begin{array}{l}\text { Plaza-Diaz et } \\
\text { al., } 2013\end{array}$ \\
\hline $\begin{array}{l}6 \text { strains Escherichia coli } \\
\left.\text { (Symbioflor }^{\odot}\right)\end{array}$ & $\begin{array}{l}1 \text { volunteer: } 2 \times 10^{8} ; \\
2 \text { volunteers: } 1 \times 10^{9} ; \\
2 \text { volunteers: } 2 \times 10^{9}\end{array}$ & $\begin{array}{l}\text { One-shot + } \\
210 \text { follow } \\
\text { up }\end{array}$ & 5 & NA & $\begin{array}{l}\text { in the observation } \\
\text { period, the mean } \\
\text { values of the probiotic } \\
E \text {. coli outnumbered } \\
\text { those of host } E \text {. coli in } \\
3 / 5 \text { subjects }\end{array}$ & $\begin{array}{l}\text { Wassenaar et } \\
\text { al., } 2014\end{array}$ \\
\hline $\begin{array}{l}4 \text { strains }(\mathrm{O} / \mathrm{C}, \mathrm{N} / \mathrm{R}, \mathrm{SIN} \\
\text { and } \mathrm{T}) \text { of Bacillus clausii } \\
\left.\text { (Enterogermina }^{\odot}\right)\end{array}$ & $\begin{array}{l}2 \times 10^{9} \text { total count of the } \\
4 \text { strains }\end{array}$ & $\begin{array}{l}\text { One-shot in } \\
\text { cross-over }\end{array}$ & 20 cross-over & $\begin{array}{l}10^{9} \text { cfu one day } \\
\text { after the one-shot } \\
\text { administration }\end{array}$ & detected till day 12 & $\begin{array}{l}\text { Ghelardi et al. } \\
2015\end{array}$ \\
\hline $\begin{array}{l}\text { L. acidophilus NCFM, B. } \\
\text { longum BB536 }\end{array}$ & $\begin{array}{l}10^{9} \text { per strain; two } \\
\text { matrices (gelatin vs } \\
\text { micro-encapsulated) }\end{array}$ & $\begin{array}{l}12+7 \text { follow } \\
\text { up }\end{array}$ & 12 & $\begin{array}{l}\text { increased recovery } \\
\text { of lactobacilli with } \\
\text { consumption of } \\
\text { encapsulated } \\
\text { probiotic }\end{array}$ & $\begin{array}{l}\text { increase lasts less than } \\
7 \text { days }\end{array}$ & $\begin{array}{l}\text { Mai et al., } \\
2017\end{array}$ \\
\hline $\begin{array}{l}\text { B. animalis subsp. lactis } \\
\text { BI-04, L. acidophilus La- } \\
\text { 14, L. plantarum SDZ-11, } \\
\text { L. paracasei SDZ-22 }\end{array}$ & $\begin{array}{l}\text { dose A }\left(7 \times 10^{9} \text { cells): }\right. \\
\text { BI-04 and La-14: } 10^{9} \text {; } \\
\text { SDZ-11: } 10^{8} ; \\
\text { SDZ-22: } 10^{7} ; \\
\text { dose B }\left(7 \times 10^{10} \text { cells): }\right. \\
\text { each strain } 10 \text { times } \\
\text { higher than in dose A }\end{array}$ & $\begin{array}{l}14+14 \\
\text { follow up }\end{array}$ & 40 & $\begin{array}{l}\text { dose } A: 15 / 20 \\
\text { positive; dose } B \text { : } \\
20 / 20 \text { positive on } \\
\text { the last day of } \\
\text { treatment }\left(10^{6}-10^{9}\right. \\
\text { cfu/g) }\end{array}$ & $\begin{array}{l}\text { only qPCR data } \\
\text { available }\end{array}$ & $\begin{array}{l}\text { Taverniti et al. } \\
2019\end{array}$ \\
\hline
\end{tabular}

\section{Matrix impact on survival and persistence}

The impact of the food matrix on survival and persistence was reported by Ghelardi et al. (2015), Saxelin et al. (2010) and Mai et al. (2017). While Ghelardi et al. (2015) reported no matrix effect, Saxelin et al. (2010) concluded that the administration of probiotic mixtures in cheese negatively affected the faecal counts of Propionibacterium spp. and Bifidobacterium spp. The possible reasons for this difference were not addressed by the authors. On the other hand, a difference between gelatine and microencapsulation, with the latter showing superior outcomes, was reported by Mai et al. (2017).

\section{Discussion}

In line with FAO/WHO statements (FAO/WHO, 2002) and Hill et al. (2014), our review highlights the importance of strain specificity in the survival and persistence of probiotic bacteria belonging to different genera: Lactobacillus, Bifidobacterium, and Bacillus. The diversity of methods employed to detect probiotic strains in faeces makes it challenging to draw direct comparisons between studies. Future studies using standardised methods and a larger sample size will offer more clarity on the dose-response relationship of probiotics. Nevertheless, an overall assessment of faecal recovery studies performed with single strain products suggests that two different groups can be identified: 
1. Recovery amount between $10^{4}$ and $10^{5} \mathrm{cfu} / \mathrm{g}$ (Arioli et al., 2018; De Champs et al., 2003; Donnet-Hughes et al., 1999; Smith et al., 2011).

2. Recovery amount higher than $10^{5} \mathrm{cfu} / \mathrm{g}$ (Ahmed et al., 2007; Alander et al., 1999; Dommels et al., 2009; Hanifi et al., 2015; Khine et al., 2019; Lund et al., 2002; Rattanaprasert et al., 2014; Saxelin et al., 1995; Vesa et al., 2000).

Multi-strain products also fall into these two categories:

1. Recovery amount between $10^{4}$ and $10^{5} \mathrm{cfu} / \mathrm{g}$ (Klingberg and Budde, 2006; Matto et al., 2006).

2. Recovery amount higher than $10^{5} \mathrm{cfu} / \mathrm{g}$ (Ghelardi et al., 2015; Jacobsen et al., 1999; Taverniti et al., 2019).

A daily dose lower than $10^{9} \mathrm{cfu}$ always resulted in a percentage of subjects testing negative for the administered strains. However, even very high doses $\left(10^{12} \mathrm{cfu}\right)$ did not lead to recovery of the probiotics in all participants (De Champs et al., 2003). This highlights the presence of healthy adults who can be termed 'non-responders' and the 'colonisation resistance' exerted by bacteria already present in their gut.

With the exception of Ahmed et al. (2007), Rochet et al. (2008) and Rattanaprasert et al. (2014), in all other singlestrain studies, the amount of probiotic recovered from faeces was lower than that administered, and ranged between $10^{3}$ and $10^{8} \mathrm{cfu}$. The Ahmed et al. (2007) study counted total bifidobacteria levels in faeces, which may account for the similar faecal counts $\left(10^{9} \mathrm{cfu}\right)$ in the high, medium and low dose groups. The Rochet et al. (2008) study used temporal temperature gradient gel electrophoresis using primers specific to the Lactobacillus-Pediococcus-LeuconostocWeissella group and real-time PCR using primers specific to the L. paracasei group, to which the administered probiotic belongs. Therefore, the faecal recovery in this study represents a general increase in gut bacteria belonging to the L. paracasei group and not a specific increase of the administered strain. In the Rattanaprasert et al. (2014) study, the amount of the probiotic strain administered was equal to that recovered from stool. However, this strain did not colonise or persist in the gut of subjects beyond the treatment period.

Three studies administered L. rhamnosus GG to subjects; of these, only one study found persistence of the administered strain after the end of treatment (Alander et al., 1999). This study compared persistence in faeces with persistence in colonic biopsy samples to show that L. rhamnosus GG persists in the gut in most subjects (7 out of 8 ) for at least a week after the end of treatment, even though it is absent from faeces. Thus, faecal recovery may underestimate persistence in studies of this strain.
Three studies treated subjects with L. reuteri DSM 17938 at $10^{8}$ or $10^{9} \mathrm{cfu}$ and recovered between $10^{5}$ and $10^{8} \mathrm{cfu}$ in faeces. Of these, only the Smith et al. (2011) study noted persistence beyond treatment in a small proportion of subjects for two weeks after treatment ended, independent of the dosage administered. This may be due to the $100 \%$ study protocol compliance noted by the authors, which may be difficult to replicate in different study settings.

Even though most strains, with the exception of L. rhamnosus GG and L. johnsonii La1 (Donnet-Hughes et al., 1999; Saxelin et al., 1995), lack a linear relationship between the administered dose and the recovered amount, a minimum dose that allows faecal recovery could be set at $10^{8} \mathrm{cfu} /$ day (Donnet-Hughes et al., 1999; Rattanaprasert et al., 2014).

For both categories of products - single strain and multistrain - the levels in stools were frequently reported to be $10^{6} \mathrm{cfu} / \mathrm{g}$ faeces. The highest amount, $10^{9} \mathrm{cfu} / \mathrm{g}$ faeces, reported by Taverniti et al. (2019) for vegetative cells, and by Hanifi et al. (2015) and Ghelardi et al. (2015) for spores, confirm that spores are highly resistant to the harsh conditions of gastrointestinal transit. When comparing the recovery rates of two different spore-formers, a higher amount (10-100 times higher) was observed for B. clausii (Ghelardi et al., 2015) than for B. subtilis RO179 (Hanifi et al., 2015). Such an effect may be species-specific rather than being related to the duration of treatment; however, further studies comparing similar treatment regimens for these species are warranted. The length of time when strains could be recovered was highly variable: it ranged from five to seven days in most studies that recorded persistence, but could also last as long as 30 or 90 days (Plaza-Diaz et al., 2013; Taverniti et al., 2019).

The selected studies contained several confounding factors. Only adults participated in these studies. The duration of treatment varied between one-shot administration and 30 days, with the majority of studies conducted over a 7 - or 14-day period. The number of participants was limited; one third of the studies enrolled ten or fewer participants. However, the overall assessment allows us to draw some conclusions.

Taken together, the dose response studies suggest that a linear relationship may exist between the administered dose and the recovered amount for specific strains. However, this is not generalisable, as there are examples in which the impact of the dose is negligible. The duration of treatment (which varies from one to thirty days) also does not affect faecal recovery or persistence (Tables 3 and 4). Dosing the same products every day or on alternate days does not influence recovery rate (Smith et al., 2011). As suggested by Taverniti and colleagues (2019), a higher dosage may increase the persistence of faecal recovery, but this may 
only apply to strains that share common characteristics, such as gastrointestinal resistance, adhesion to the mucosa, and ability to multiply in the gut.

Different Lactobacillus sp. have different minimum doses that allow faecal recovery: $10^{8} \mathrm{cfu} /$ day for L. reuteri DSM 17938 and $10^{9}$ for L. rhamnosus GG. This dose effect is apparent up to $10^{10} \mathrm{cfu} /$ day, with strain-dependent variations related to the amount administered. For doses larger than $10^{10} \mathrm{cfu} /$ day, faecal recovery appears to be independent from dosage. The highest faecal recovery reported was $10^{9} \mathrm{cfu} / \mathrm{g}$. These data suggest a dose response effect, as reported by Ouwehand et al. (2017). However, the results of this analysis also confirm that strains may differ in their ability to survive the gastrointestinal tract and to proliferate in the gut. The dosage and the treatment duration appear to play a secondary role. Similar conclusions apply to Bifidobacterium and other species, in spite of the lower number of studies involving these species.

\section{Conclusions}

Faecal recovery and persistence in the gut are straindependent, but doses higher than $10^{10} \mathrm{cfu} /$ day generate a good level of faecal recovery independent of the strain. Faecal recovery and persistence are independent of treatment duration. The food matrix may affect the survival of probiotics, but the number of studies that test this factor are too few to allow us to draw conclusions. In summary, despite variability across strains, current data suggest that the dose required for the majority of strains to survive and persist are higher than the doses recommended for probiotics by regulatory agencies.

\section{Acknowledgements}

Editorial support, including language editing and formatting, was provided by Subhashini Muralidharan, $\mathrm{PhD}$, of inScience Communications, Springer Healthcare Ltd, UK, and was funded by Sanofi.

\section{References}

Ahmed, M., Prasad, J., Gill, H., Stevenson, L. and Gopal, P., 2007. Impact of consumption of different levels of Bifidobacterium lactis HN019 on the intestinal microflora of elderly human subjects. Journal of Nutrition, Health and Aging 11: 26-31.

Alander, M., Satokari, R., Korpela, R., Saxelin, M., Vilpponen-Salmela, T., Mattila-Sandholm, T. and Von Wright, A., 1999. Persistence of colonization of human colonic mucosa by a probiotic strain, Lactobacillus rhamnosus GG, after oral consumption. Applied and Environmental Microbiology 65: 351-354. https://doi.org/10.1128/ AEM.65.1.351-354.1999
Arioli, S., Koirala, R., Taverniti, V., Fiore, W. and Guglielmetti, S., 2018. Quantitative recovery of viable Lactobacillus paracasei $\mathrm{CNCM}$ I-1572 (L. casei DG(R)) after gastrointestinal passage in healthy adults. Frontiers in Microbiology 9: 1720. https://doi.org/10.3389/ fmicb.2018.01720

CIRS, 2019. Current situation of regulations and application requirements on probiotic health food in China. CIRS, Hangzhou, China. Available at: https://tinyurl.com/xwt3wfu6

De Champs, C., Maroncle, N., Balestrino, D., Rich, C. and Forestier, C., 2003. Persistence of colonization of intestinal mucosa by a probiotic strain, Lactobacillus casei subsp. rhamnosus Lcr35, after oral consumption. Journal of Clinical Microbiology 41: 1270-1273. https://doi.org/10.1128/jcm.41.3.1270-1273.2003

Dommels, Y.E., Kemperman, R.A., Zebregs, Y.E., Draaisma, R.B., Jol, A., Wolvers, D.A., Vaughan, E.E. and Albers, R., 2009. Survival of Lactobacillus reuteri DSM 17938 and Lactobacillus rhamnosus GG in the human gastrointestinal tract with daily consumption of a low-fat probiotic spread. Applied and Environmental Microbiology 75: 6198-6204. https://doi.org/10.1128/AEM.01054-09

Donnet-Hughes, A., Rochat, F., Serrant, P., Aeschlimann, J.M. and Schiffrin, E.J., 1999. Modulation of nonspecific mechanisms of defense by lactic acid bacteria: effective dose. Journal of Dairy Science 82: 863-869. https://doi.org/10.3168/jds.S00220302(99)75304-X

European Food Safety Authority (EFSA), 2016. Outcome of a public consultation of the EFSA Panel on Dietetic Products, Nutrition and Allergies (NDA) on the draft general scientific guidance for stakeholders on health claim applications. EFSA Supporting Publications 13(1): 986E. https://doi.org/10.2903/sp.efsa.2016. EN-986

Food and Agriculture Organisation / World Health Organisation (FAO/ WHO), 2001. Report of a joint FAO/WHO expert consultation on evaluation of health and nutritional properties of probiotics in food including powder milk with live lactic acid bacteria. FAO/WHO, Rome/Geneva, Italy/Switzerland. Available at: http://www.fao. org/3/a0512e/a0512e.pdf

Food and Agriculture Organisation / World Health Organisation (FAO/WHO), 2002. Guidelines for the evaluation of probiotics in food. FAO/WHO, Rome/Geneva, Italy/Switzerland. Available at: https://www.who.int/foodsafety/fs_management/en/probiotic_ guidelines.pdf

Ghelardi, E., Celandroni, F., Salvetti, S., Gueye, S.A., Lupetti, A. and Senesi, S., 2015. Survival and persistence of Bacillus clausii in the human gastrointestinal tract following oral administration as sporebased probiotic formulation. Journal of Applied Microbiology 119: 552-559. https://doi.org/10.1111/jam.12848

Hanifi, A., Culpepper, T., Mai, V., Anand, A., Ford, A.L., Ukhanova, M., Christman, M., Tompkins, T.A. and Dahl, W.J., 2015. Evaluation of Bacillus subtilis R0179 on gastrointestinal viability and general wellness: a randomised, double-blind, placebo-controlled trial in healthy adults. Beneficial Microbes 6: 19-27. https://doi.org/10.3920/ BM2014.0031 
Hill, C., Guarner, F., Reid, G., Gibson, G.R., Merenstein, D.J., Pot, B., Morelli, L., Canani, R.B., Flint, H.J., Salminen, S., Calder, P.C. and Sanders, M.E., 2014. Expert consensus document. The International Scientific Association for Probiotics and Prebiotics consensus statement on the scope and appropriate use of the term probiotic. Nature Reviews: Gastroenterology and Hepatology 11: 506-514. https://doi.org/10.1038/nrgastro.2014.66

Hutt, P., Koll, P., Stsepetova, J., Alvarez, B., Mandar, R., KroghAndersen, K., Marcotte, H., Hammarstrom, L. and Mikelsaar, M., 2011. Safety and persistence of orally administered human Lactobacillus sp. strains in healthy adults. Beneficial Microbes 2: 79-90. https://doi.org/10.3920/BM2010.0023

Jacobsen, C.N., Rosenfeldt Nielsen, V., Hayford, A.E., Moller, P.L., Michaelsen, K.F., Paerregaard, A., Sandstrom, B., Tvede, M. and Jakobsen, M., 1999. Screening of probiotic activities of forty-seven strains of Lactobacillus spp. by in vitro techniques and evaluation of the colonization ability of five selected strains in humans. Applied and Environmental Microbiology 65: 4949-4956. https://doi. org/10.1128/AEM.65.11.4949-4956.1999

Khine, W.W.T., Ang, X.J., Chan, Y.S., Lee, W.Q., Quek, S.Y., Tan, S.H., Teo, H.T.A., Teo, J.K.B., Lau, Q.C. and Lee, Y.K., 2019. Recovery of Lactobacillus casei strain Shirota (LcS) from faeces of healthy Singapore adults after intake of fermented milk. Beneficial Microbes 10: 721-728. https://doi.org/10.3920/BM2018.0173

Klingberg, T.D. and Budde, B.B., 2006. The survival and persistence in the human gastrointestinal tract of five potential probiotic lactobacilli consumed as freeze-dried cultures or as probiotic sausage. International Journal of Food Microbiology 109: 157-159. https://doi.org/10.1016/j.ijfoodmicro.2006.01.014

Larsen, C.N., Nielsen, S., Kaestel, P., Brockmann, E., Bennedsen, M., Christensen, H.R., Eskesen, D.C., Jacobsen, B.L. and Michaelsen, K.F., 2006. Dose-response study of probiotic bacteria Bifidobacterium animalis subsp lactis BB-12 and Lactobacillus paracasei subsp paracasei CRL-341 in healthy young adults. European Journal of Clinical Nutrition 60: 1284-1293. https://doi.org/10.1038/ sj.ejcn. 1602450

Lund, B., Adamsson, I. and Edlund, C., 2002. Gastrointestinal transit survival of an Enterococcus faecium probiotic strain administered with or without vancomycin. International Journal of Food Microbiology 77: 109-115. https://doi.org/10.1016/s01681605(02)00047-8

Mai, V., Waugh, S., Byrd, D., Simpson, D. and Ukhanova, M., 2017. Novel encapsulation improves recovery of probiotic strains in fecal samples of human volunteers. Applied Microbiology and Biotechnology 101: 1419-1425. https://doi.org/10.1007/s00253016-7915-8

Matto, J., Fonden, R., Tolvanen, T., Von Wright, A., VilpponenSalmela, T., Satokari, R. and Saarela, M., 2006. Intestinal survival and persistence of probiotic Lactobacillus and Bifidobacterium strains administered in triple-strain yoghurt. International Dairy Journal 16: 1174-1180. https://doi.org/10.1016/j.idairyj.2005.10.007 Ministero della Salute, 2013. Guidelines on probiotics and prebiotics. Ministero della Salute, Rome, Italy. Available at: https://tinyurl. com/234ecjfa
Ouwehand, A.C., 2017. A review of dose-responses of probiotics in human studies. Beneficial Microbes 8: 143-151. https://doi. org/10.3920/BM2016.0140

Plaza-Diaz, J., Gomez-Llorente, C., Campana-Martin, L., Matencio, E., Ortuno, I., Martinez-Silla, R., Gomez-Gallego, C., Periago, M.J., Ros, G., Chenoll, E., Genoves, S., Casinos, B., Silva, A., Corella, D., Portoles, O., Romero, F., Ramon, D., Perez de la Cruz, A., Gil, A. and Fontana, L., 2013. Safety and immunomodulatory effects of three probiotic strains isolated from the feces of breast-fed infants in healthy adults: SETOPROB study. PLoS ONE 8: e78111. https:// doi.org/10.1371/journal.pone.0078111

Prilassnig, M., Wenisch, C., Daxboeck, F. and Feierl, G., 2007. Are probiotics detectable in human feces after oral uptake by healthy volunteers? Wiener Klinische Wochenschrift 119: 456-462. https:// doi.org/10.1007/s00508-007-0808-1

Rattanaprasert, M., Roos, S., Hutkins, R.W. and Walter, J., 2014. Quantitative evaluation of synbiotic strategies to improve persistence and metabolic activity of Lactobacillus reuteri DSM 17938 in the human gastrointestinal tract. Journal of Functional Foods 10: 85-94. https://doi.org/10.1016/j.jff.2014.05.017

Rochet, V., Rigottier-Gois, L., Levenez, F., Cadiou, J., Marteau, P., Bresson, J.L., Goupil-Feillerat, N. and Dore, J., 2008. Modulation of Lactobacillus casei in ileal and fecal samples from healthy volunteers after consumption of a fermented milk containing Lactobacillus casei DN-114 001Rif. Canadian Journal of Microbiology 54: 660667. https://doi.org/10.1139/w08-050

Sánchez, B., Delgado, S., Blanco-Míguez, A., Lourenço, A., Gueimonde, M. and Margolles, A., 2017. Probiotics, gut microbiota, and their influence on host health and disease. Molecular Nutrition and Food Research 61: 1600240. https://doi.org/10.1002/mnfr.201600240

Sanders, M.E., Merenstein, D.J., Reid, G., Gibson, G.R. and Rastall, R.A., 2019. Probiotics and prebiotics in intestinal health and disease: from biology to the clinic. Nature Reviews Gastroenterology and Hepatology 16: 605-616. https://doi.org/10.1038/s41575-019-0173-3

Saxelin, M., Lassig, A., Karjalainen, H., Tynkkynen, S., Surakka, A., Vapaatalo, H., Jarvenpaa, S., Korpela, R., Mutanen, M. and Hatakka, K., 2010. Persistence of probiotic strains in the gastrointestinal tract when administered as capsules, yoghurt, or cheese. International Journal of Food Microbiology 144: 293-300. https:// doi.org/10.1016/j.ijfoodmicro.2010.10.009

Saxelin, M., Pessi, T. and Salminen, S., 1995. Fecal recovery following oral-administration of Lactobacillus strain Gg (Atcc-53103) in gelatin capsules to healthy-volunteers. International Journal of Food Microbiology 25: 199-203. https://doi.org/10.1016/01681605(94)00091-J

Shinoda, T., Kusuda, D., Ishida, Y., Ikeda, N., Kaneko, K., Masuda, O. and Yamamoto, N., 2001. Survival of Lactobacillus helveticus strain CP53 in the human gastrointestinal tract. Letters in Applied Microbiology 32: 108-113. https://doi.org/10.1046/j.1472765x.2001.00870.x

Smith, T.J., Anderson, D., Margolis, L.M., Sikes, A. and Young, A.J., 2011. Persistence of Lactobacillus reuteri DSM17938 in the human intestinal tract: response to consecutive and alternate-day supplementation. Journal of the American College of Nutrition 30: 259-264. https://doi.org/10.1080/07315724.2011.10719968 
Szajewska, H. and Hojsak, I., 2020. Health benefits of Lactobacillus rhamnosus GG and Bifidobacterium animalis subspecies lactis BB-12 in children. Postgraduate Medicine 132: 441-451. https:// doi.org/10.1080/00325481.2020.1731214

Taverniti, V., Koirala, R., Dalla Via, A., Gargari, G., Leonardis, E., Arioli, S. and Guglielmetti, S., 2019. Effect of cell concentration on the persistence in the human intestine of four probiotic strains administered through a multispecies formulation. Nutrients 11: 285. https://doi.org/10.3390/nu11020285

Van Bokhorst-Van de Veen, H., Van Swam, I., Wels, M., Bron, P.A. and Kleerebezem, M., 2012. Congruent strain specific intestinal persistence of Lactobacillus plantarum in an intestine-mimicking in vitro system and in human volunteers. PLoS ONE 7: e44588. https://doi.org/10.1371/journal.pone.0044588

Vesa, T., Pochart, P. and Marteau, P., 2000. Pharmacokinetics of Lactobacillus plantarum NCIMB 8826, Lactobacillus fermentum KLD, and Lactococcus lactis MG 1363 in the human gastrointestinal tract. Alimentary Pharmacology and Therapeutics 14: 823-828. https://doi.org/10.1046/j.1365-2036.2000.00763.x
Wassenaar, T.M., Beimfohr, C., Geske, T. and Zimmermann, K., 2014. Voluntarily exposure to a single, high dose of probiotic Escherichia coli results in prolonged colonisation. Beneficial Microbes 5: $367-$ 375. https://doi.org/10.3920/BM2013.0087

Zheng, J., Wittouck, S., Salvetti, E., Franz, C., Harris, H.M.B., Mattarelli, P., O’Toole, P.W., Pot, B., Vandamme, P., Walter, J., Watanabe, K., Wuyts, S., Felis, G.E., Ganzle, M.G. and Lebeer, S., 2020. A taxonomic note on the genus Lactobacillus: description of 23 novel genera, emended description of the genus Lactobacillus Beijerinck 1901, and union of Lactobacillaceae and Leuconostocaceae. International Journal of Systematic and Evolutionary Microbiology 70: 2782-2858. https://doi.org/10.1099/ijsem.0.004107

Zorzela, L., Ardestani, S.K., McFarland, L.V. and Vohra, S., 2017. Is there a role for modified probiotics as beneficial microbes: a systematic review of the literature. Beneficial Microbes 8: 739-754. https://doi.org/10.3920/BM2017.0032 
